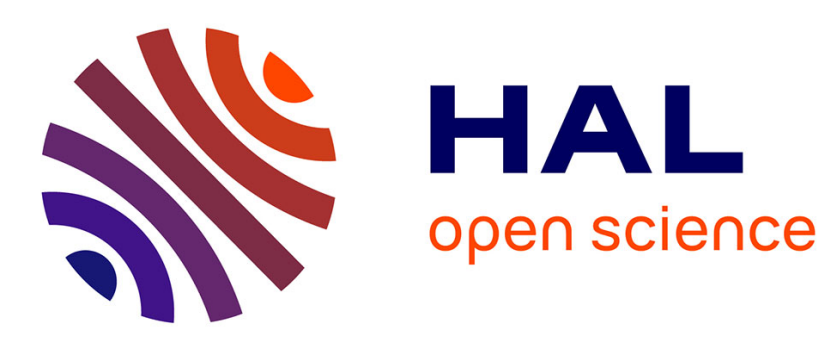

\title{
Interactions of spatiotemporal solitons and vortices in fiber bundles
}

\author{
Hervé Leblond, Boris Malomed, Dumitru Mihalache
}

\section{To cite this version:}

Hervé Leblond, Boris Malomed, Dumitru Mihalache. Interactions of spatiotemporal solitons and vortices in fiber bundles. Physical Review A : Atomic, molecular, and optical physics [1990-2015], 2009, 79 (3), pp.033841. 10.1103/PhysRevA.79.033841 . hal-03423762

\section{HAL Id: hal-03423762 \\ https://univ-angers.hal.science/hal-03423762}

Submitted on 10 Nov 2021

HAL is a multi-disciplinary open access archive for the deposit and dissemination of scientific research documents, whether they are published or not. The documents may come from teaching and research institutions in France or abroad, or from public or private research centers.
L'archive ouverte pluridisciplinaire HAL, est destinée au dépôt et à la diffusion de documents scientifiques de niveau recherche, publiés ou non, émanant des établissements d'enseignement et de recherche français ou étrangers, des laboratoires publics ou privés. 


\title{
Interactions of spatiotemporal solitons and vortices in fiber bundles
}

\author{
Hervé Leblond, ${ }^{1}$ Boris A. Malomed, ${ }^{2}$ and Dumitru Mihalache ${ }^{3,4}$ \\ ${ }^{1}$ Laboratoire POMA, FRE 2988, Université d'Angers, 2 Bd Lavoisier, 49000 Angers, France \\ ${ }^{2}$ Department of Physical Electronics, School of Electrical Engineering, Faculty of Engineering, \\ Tel Aviv University, Tel Aviv 69978, Israel \\ ${ }^{3}$ Department of Theoretical Physics, Horia Hulubei National Institute for Physics and Nuclear Engineering (IFIN-HH), \\ 407 Atomistilor, Magurele-Bucharest 077125, Romania \\ ${ }^{4}$ Academy of Romanian Scientists, 54 Splaiul Independentei, Bucharest 050094, Romania
}

(Received 4 February 2009; published 26 March 2009)

\begin{abstract}
We report results of a systematic numerical analysis of collisions between localized three-dimensional (3D) semidiscrete complexes, viz., rhombus-shaped vortices, quadrupoles, and fundamental solitons, in the model of a bundle of fiberlike waveguides. The model also describes a 3D self-attractive Bose-Einstein condensate (BEC) loaded into a deep two-dimensional optical lattice. We identify four outcomes of the collisions: rebound of slow solitons, fusion, splitting, and, finally, quasielastic interactions of fast solitons. Diagrams which demonstrate regions of the different outcomes are reported for collisions between vortices, with equal or opposite topological charges, and quadrupoles.
\end{abstract}

DOI: 10.1103/PhysRevA.79.033841 PACS number(s): 42.65.Tg, 42.81.Dp, 03.75.Lm, 05.45.Yv

\section{INTRODUCTION}

Studies of discrete spatial solitons (alias lattice solitons) attract a great deal of interest in the current experimental and theoretical works in the fields of photonic and matter-wave optics [1]. A fundamental model of lattice media amounts to the discrete nonlinear Schrödinger (DNLS) equation [2]. A direct realization of the one-dimensional (1D) DNLS model with the cubic nonlinearity in arrays of optical waveguides was first proposed in Ref. [3]. Later, the same DNLS equation was demonstrated to describe, in various theoretical and experimental settings, Bose-Einstein condensates (BECs) trapped in deep optical-lattice potentials (see original works [4] and Ref. [5] for a review). Another physical realization of the DNLS model is known in the form of lattices built of polariton-trapping microcavities [6]. Discrete solitons in 1D optical structures were created experimentally in a set of semiconductor waveguides built on a slab substrate [7] and then in arrays of optical fibers [8]. Apart from such permanent structures, waveguiding arrays have also been developed in a virtual form as optically induced lattices in photorefractive crystals [9]. The optoinduction technique has helped to create quasidiscrete two-dimensional (2D) solitons too [10]. Vortex solitons, i.e., localized lattice excitations with embedded vorticity, that were predicted in Ref. [11] were also created in 2D virtual photonic lattices [12]. In addition to the fundamental discrete vortices with topological charge $S= \pm 1$, higher-order solitary vortices, with $|S|>1$, and multipole solitons, such as quadrupoles, were predicted in Refs. [13,14]. Supervortices (ring-shaped chains of compact vortices carrying a global vorticity, which is independent of the vorticity of the individual elements [14]), lattice solitons in the second band gap [15], necklace-shaped localized patterns [16], discrete solitons in hexagonal and triangular lattices [17], and semidiscrete composite solitons in arrays of quadratically nonlinear waveguides and in arrayed waveguide structures with the Kerr nonlinearity [18] were studied too, in the theoretical and experimental forms, as well as nonstationary effects, including the mobility of discrete solitons $[19,20]$, collisions between them $[20,21]$, and the onset of the spatiotemporal collapse in arrays of selffocusing waveguides [22].

Continuing this line of the research, the creation of $2 \mathrm{D}$ spatial solitons was recently reported [23] in a bundle of fiberlike waveguides permanently written in bulk silica using the technique based on tightly focused femtosecond laser pulses [24]. Similarly structured quasidiscrete 2D solitons, including solitary vortices, were predicted in photoniccrystal fibers [25].

In the above-mentioned settings, the work was chiefly focused on the spatial-domain dynamics. In particular, because of a very large response time in photorefractive crystals, optically induced lattices do not allow one to study the dynamics in the temporal domain. Nevertheless, the settings based on fiber bundles, or arrays of waveguides written in bulk silica, feature a very fast response to variations of the light beams, which suggests a possibility to study the spatiotemporal dynamics combining the temporal evolution in the longitudinal direction and quasidiscrete spatial patterns in the transverse direction (see Ref. [26] for a review of the topic of spatiotemporal solitons in nonlinear optics and BEC). Various manifestations of the nonlinear behavior have been studied in this context, including semidiscrete "light bullets" (spatiotemporal solitons) in arrays or fibers [27] and photonic wires [28], self-compression [29] and steering [30] of pulsed beams, and the modulational instability [31]. Spatiotemporal solitons in models of waveguide arrays with the quadratic (rather than cubic) nonlinearity were studied too [32].

Continuing the work in this direction, semidiscrete spatiotemporal surface solitons were recently introduced in semi-infinite models of waveguide arrays [33] and in a system with an interface between two different arrays [34]. In particular, solitons of a combined staggered-unstaggered type [35] have been reported in the latter case.

The above-mentioned experimental techniques, which allow one to create solitary modes in bundled arrays of fiber- 
like waveguides [23,24], suggests the consideration of semidiscrete three-dimensional (3D) spatiotemporal solitons as localized states which are continuous along the propagation axis and discrete in the transverse plane. The same solutions should be relevant to the description of 3D matter-wave solitons in a self-attractive BEC trapped in a deep 2D optical lattice [36]. The latter configuration can be created by shining a "bundled" set of parallel narrow red-detuned (attractive) laser beams through the 3D condensate.

In recent work [37], we have reported results of a systematic analysis of spatiotemporal vortex solitons and quadrupoles in the semidiscrete 3D model. It was demonstrated that solitary vortices (with $|S|=1$ ) and quadrupoles, in the form of rhombuses which are based on a set of four guiding cores, with an almost empty one at the center, have a vast stability region. Solitary vortices of the "square" type, without an empty site in the middle, feature a much smaller stability region and all vortices with $|S|=2$ are unstable (fundamental temporal solitons, which are carried, essentially, by a single lattice site, are stable). Related analyses were reported for vortex solitons with topological charges $|S|=1$ and $|S|=2$ in the continuum counterpart of the model, which includes a $2 \mathrm{D}$ periodic potential in the transverse plane [38]. It is also relevant to mention that families of discrete vortex solitons in the full DNLS equation in three dimensions were reported in Ref. [39].

Once stable soliton complexes with the topological structure have been found, an issue of straightforward interest is to consider collisions between them, which is the subject of the present work. We focus on the systematic analysis of collisions between solitons of the most robust types, i.e., rhombic vortices and quadrupoles, including collisions between the vortices with topological charges $\left(S_{1}, S_{2}\right)=(+1$, $+1)$ and $(+1,-1)$ ("corotating" and "counter-rotating" vortex pairs), as well as collisions between vortices and quadrupoles. In fact, this is an example of a 3D conservative model which makes it possible to study collisions between vortex solitons in three dimensions. Previously (in fact, very recently), collisions between coaxial 3D solitons with embedded vorticities were studied in the framework of the continual complex Ginzburg-Landau equation with the cubicquintic nonlinearity for both corotating [40] and counterrotating [41] configurations. In this work, we also consider collisions between the rhombus-shaped vortex and a fundamental soliton carried by the central waveguide of the bundle, which is nearly empty in the vortex state.

The semidiscrete structure of the rhombuses and quadrupoles helps to clearly identify four different outcomes of the collisions. With the gradual increase of the collision velocity, we first observe a rebound of the solitons due to repulsion between them, which is changed by splitting, fusion, and, eventually, quasielastic passage of the colliding complexes through each other at largest values of the velocity. For symmetric collisions between vortices and quadrupoles, the results are summarized in the form of respective diagrams which display the outcome as a function of the collision velocity and the solitons' propagation constant.

The paper is structured as follows. The model and basic types of the stable solitons are presented in Sec. II. Results for collisions between two rhombic vortices (for both co- and counter-rotating pairs), as well as collisions between quadrupoles, are reported in Sec. III. Collisions between solitons of different types (vortex-quadrupole and vortex-fundamental solitons) are considered in Sec. IV. The paper is concluded by Sec. V.

\section{SEMIDISCRETE MODEL AND SOLITONS}

Following Ref. [37], the evolution equations for local amplitudes $u_{m, n}$ of the electromagnetic waves in the bundle of fiber waveguides, whose cross section is a square grid with discrete coordinates $m$ and $n$, are taken as

$$
\begin{aligned}
\left(i \frac{\partial}{\partial z}\right. & \left.+\frac{1}{2} \frac{\partial^{2}}{\partial \tau^{2}}+\left|u_{m, n}\right|^{2}\right) u_{m, n}+\left(u_{m+1, n}+u_{m-1, n}+u_{m, n+1}\right. \\
& \left.+u_{m, n-1}-4 u_{m, n}\right)=0 .
\end{aligned}
$$

Here, $z$ is the propagation distance along the waveguides and $\tau$ the usual reduced time, while the respective coefficient of the anomalous group-velocity dispersion [42] and the constant accounting for the transverse coupling between adjacent waveguiding cores are both scaled to be 1 (in physical units, the coupling length in bundled arrays of waveguides written in bulk silica is estimated to be on the order of several centimeters $[23,24]$, with the separation between waveguides $\sim 20-50 \mu \mathrm{m}$ ).

3D BEC trapped in a deep 2D optical lattice is described by a system of linearly coupled 1D Gross-Pitaevskii equations for the wave functions in "potential tubes" induced by the lattice. In a properly scaled form, the coupled system is tantamount to Eq. (1), with $z$ and $\tau$ replaced by time and the longitudinal coordinate, respectively.

Equation (1) conserves three dynamical invariants: the Hamiltonian, momentum in the $\tau$ direction, and energy (or the number of atoms, in the case of BEC)

$$
E=\sum_{m, n} \int_{-\infty}^{+\infty}\left|u_{m, n}(\tau)\right|^{2} d \tau .
$$

Stationary solutions to Eq. (1) are parameterized by real propagation constant $\mu$ (alias the chemical potential, in the case of the BEC), $u_{m, n}(z, \tau)=e^{i \mu z} U_{m, n}(\tau)$, with complex functions $U_{m, n}$ obeying a system of ordinary differential equations

$$
\begin{aligned}
& \frac{1}{2} \frac{d^{2} U_{m, n}}{d \tau^{2}}+\left|U_{m, n}\right|^{2} U_{m, n}+\left(U_{m+1, n}+U_{m-1, n}+U_{m, n+1}+U_{m, n-1}\right) \\
& =(4+\mu) U_{m, n} .
\end{aligned}
$$

For continuous-wave (cw, i.e., $\tau$-independent) solutions, Eq. (3) reduces to coupled algebraic equations, whose solutions for vortices with topological charge $S= \pm 1$ were found, in a numerical form, in Ref. [11] and their higher-order counterparts, with $S=2$ and 3, were constructed in Ref. [13].

As well as in the case of their 2D discrete counterparts, there are two distinct types of fully localized 3D semidiscrete vortex and quadrupole solitons, viz., rhombuses and squares. As said above, in this work we focus on vortical rhombuses, with $|S|=1$, and rhombic quadrupoles, which are far more 
robust than squares [37]. The frame of the rhombus is composed of four vertices, with coordinates

$$
(m, n)=(1,0),(0,1),(-1,0),(0,-1),
$$

while the central site, at $(m, n)=(0,0)$, remains nearly empty in the rhombic states. Solutions to Eq. (3) in the form of rhombus-shaped vortices were constructed in Ref. [37] using a combination of numerical methods and the variational approximation (VA). The latter was based on ansatz

$$
\left(U_{m, n}\right)_{\text {rhom }}=A(m+i n) e^{-a(|m|+|n|)} \operatorname{sech}(\eta \tau) .
$$

In the case of narrow vortices [those strongly confined to frame (4), which corresponds to $\mu \gtrsim 1$; in fact, the stability region for the rhombic vortices is $\mu \gtrsim 19$ [37], hence they are definitely narrow ones], parameters $A, a$, and $\eta$ of the ansatz, which determine that amplitude, transverse extension, and longitudinal size of the semidiscrete vortex, are predicted by the VA to be

$$
\eta^{2}=2(4+\mu), \quad e^{a}=2(4+\mu), \quad A^{2}=[2(4+\mu)]^{3} .
$$

In the same approximation, energy (2) of the rhombic vortex is

$$
E=8 \sqrt{2(4+\mu)} .
$$

Another robust species of the 2D semidiscrete solitons, in the form of quadrupoles, is also based on "frame" (4). Approximate solutions for the quadrupoles (in addition to numerical solutions) were constructed in Ref. [37] using the following real variational ansatz, cf. its complex counterpart (5):

$$
\left(U_{m, n}\right)_{\text {quad }}=A\left(m^{2}-n^{2}\right) e^{-a(|m|+|n|)} \operatorname{sech}(\eta \tau) .
$$

For narrow quadrupoles, the VA predicts

$$
\eta^{2}=2(4+\mu), \quad e^{a}=\frac{16}{3}(4+\mu), \quad A^{2}=\frac{1}{9}[8(4+\mu)]^{3} .
$$

cf. Eq. (6). The stability region for the quadrupoles is $\mu$ $\geq 20$ [37], which means that they are narrow too. In the first approximation, the energy of the quadrupole coincides with that given by expression (7) for the vortices. Comparison of the variational results given by Eqs. (5)-(9) demonstrates that the VA is very accurate for the stable vortices and quadrupoles alike.

In view of the Galilean invariance of Eq. (1), a moving solution can be generated from any quiescent one, such as vortex [Eq. (5)] or quadrupole [Eq. (8)], by the application of the boost, with arbitrary velocity parameter $c$,

$$
u_{m, n}(t ; c)=\exp \left(i c \tau-i c^{2} z / 2\right) u(t-c z ; 0) .
$$

The moving solitons will be used below as initial configurations for simulations of collisions between them.

\section{COLLISIONS BETWEEN IDENTICAL SOLITONS}

Results for outcomes of collisions between solitons were collected by systematic simulations of Eq. (1). Two solitons

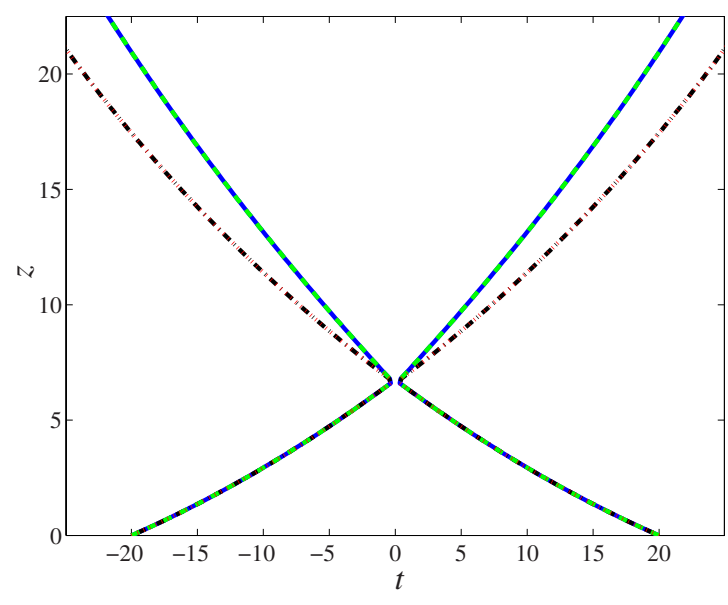

FIG. 1. (Color online) An example of the quasielastic collision of two identical rhombus-shaped vortices, with topological charges $S_{1}=S_{2}=1$, propagation constants $\mu_{1}=\mu_{2}=20$, and relative velocity $\Delta v=8$. Collision-induced changes in the configurations are shown by trajectories of the motion of four fundamental solitons that constitute each vortex. In this figure and below, solid blue, dasheddotted black, dashed green, and dotted red curves designate the trajectories of the fundamental solitons belonging to waveguiding cores with the coordinates, respectively, $(-1,0),(0,-1),(1,0)$, and $(0,1)$ [cf. Eq. (4)].

with a large initial temporal separation between them were set in motion with relative velocity $\Delta V$ by means of boost (10) with $c_{1,2}= \pm \Delta V / 2$. The initial shapes of the rhombuses and quadrupoles were taken as per the VA prediction, i.e., in the form of Eq. (5) and (9). As mentioned above, for stable solitons the VA yields a practically exact shape of stationary solutions, whose small deviations from the numerically exact ones produce a negligible perturbation.

\section{A. Collisions between corotating rhombic vortices}

We start by presenting systematic results for collisions between "corotating" rhombus-shaped vortices, i.e., ones with equal topological charges, $S_{1}=S_{2}=1$. Collisions between the vortices with a large relative velocity naturally lead to their passage through each other. The passage is quasielastic, but not always completely elastic. As seen in the example shown in Fig. 1, the collision gives rise to a slowly developing splitting of each rhombus into two pairs of fundamental solitons located at opposite vertices of the rhombus (in this case, we show only trajectories of the fundamentalsoliton components which build the vortices; full collision pictures, that show the shape of the solitons, are displayed below in Figs. 3 and 6, where the additional illustrations are necessary in view of a rather complex character of the interactions). The quasielastic passage does not break the phase structure of the vortex configuration, which, for vorticity $|S|=1$, implies the phase shift of $\pi / 2$ between adjacent vertices, hence each of the two soliton pairs, into which the rhombus slowly splits after the quasielastic collision, is actually a dipole, with opposite signs of its two constituents.

At smaller velocities or larger values of the energy of the vortices [i.e., larger $\mu$, according to Eq. (7)], the collision 


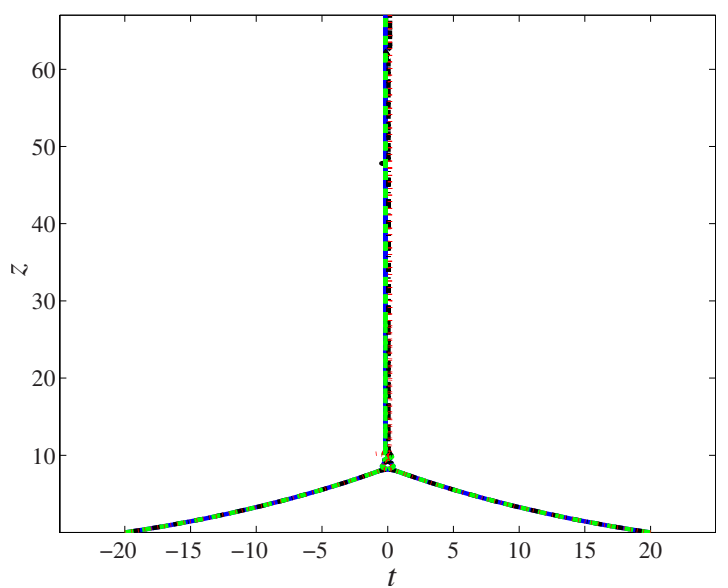

FIG. 2. (Color online) An example of merger (fusion) resulting from the collision of identical rhombic vortices, with propagation constants $\mu=22$ and relative velocity $\Delta V=8$.

becomes inelastic, leading to a merger (fusion) of the vortices into a single one of the same type as shown in Fig. 2. The further increase of $\mu$, or decrease of the collision velocity, makes the collision still more inelastic and more destructive. In this case, the collisions seem as in Fig. 3 (which displays a collision between counter-rotating rhombus-shaped vortices). In Fig. 3, one observes that the colliding rhombuses split into a pair of two-soliton bound states, which stay put after the collision, and four separate fundamental solitons, three of which escape, while another one stays together with the two-soliton bound states. Analysis of the numerical results demonstrates that this collision destroys the original phase structure of the vortices. Moreover, the collision clearly manifests spontaneous symmetry breaking as the collision of two mutually symmetric vortices gives rise to an asymmetric picture. The latter effect can probably be explained as a result of an intrinsic instability developing in the overlapping soliton cluster in the course of the collision.

The character of the interaction demonstrates another abrupt change at essentially smaller values of the relative

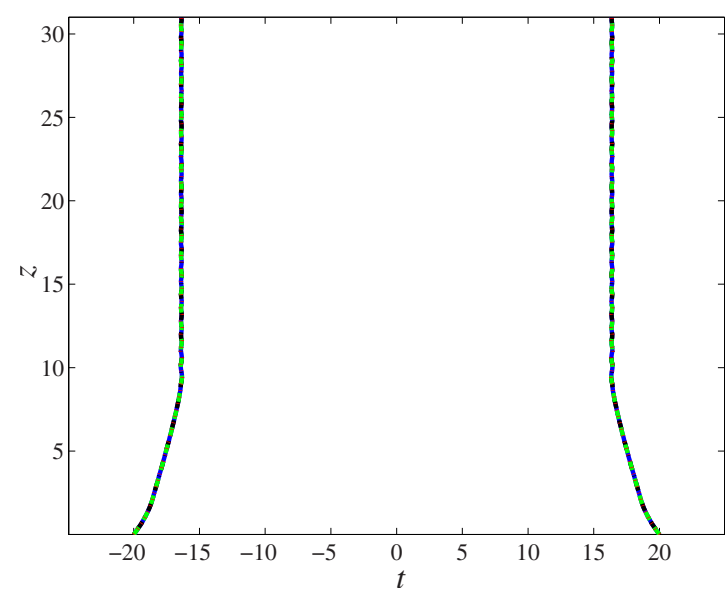

FIG. 4. (Color online) An example of the collision between two identical slowly moving rhombic vortices, with relative velocity $\Delta V=2$ and propagation constant $\mu=22$. Due to the repulsive interaction, both vortices come to a halt.

velocity: as seen in Fig. 4, repulsion between slowly moving vortices actually prevents the collision. As a result, both vortices come to a halt, keeping a large temporal distance between them. Additional analysis of numerical data demonstrates that the intrinsic phase structure of the vortices is not destroyed by the interaction, in this case.

Results of a large number of simulation runs are summarized in the diagram presented in Fig. 5 in the plane of $(\Delta V, \mu)$. It shows borders between regions corresponding to the four different outcomes of the collision, which are specified by means of the above examples.

\section{B. Collisions between counter-rotating rhombic vortices}

We have also investigated collisions between "counterrotating" rhombus-shaped vortices, i.e., ones with opposite topological charges $S_{1}=+1$ and $S_{2}=-1$. In this case, the results are quite similar to those reported above for $S_{1}=S_{2}=1$ (see Fig. 3) and the respective collision diagram is virtually
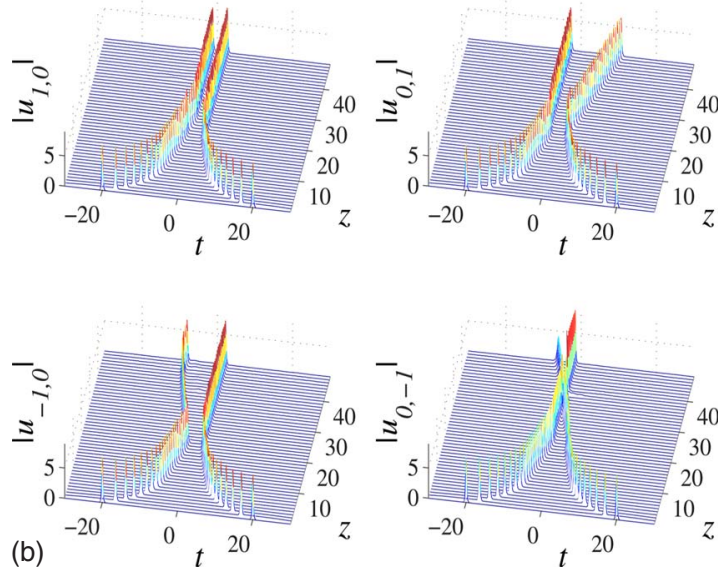

FIG. 3. (Color online) An example of splitting of two colliding rhombic vortices, with opposite topological charges, $S_{1}=-S_{2}=1$, propagation constants $\mu_{1}=\mu_{2}=25$, and relative velocity $\Delta V=8$. In addition to trajectories of fundamental solitons that constitute the vortices (a), panel (b) displays the full evolution picture in terms of $\left|u_{m n}(\tau, x)\right|$ in four waveguiding cores which carry the vortices. It is seen that the collision between identical vortices results in the spontaneous symmetry breaking, in the present case. 


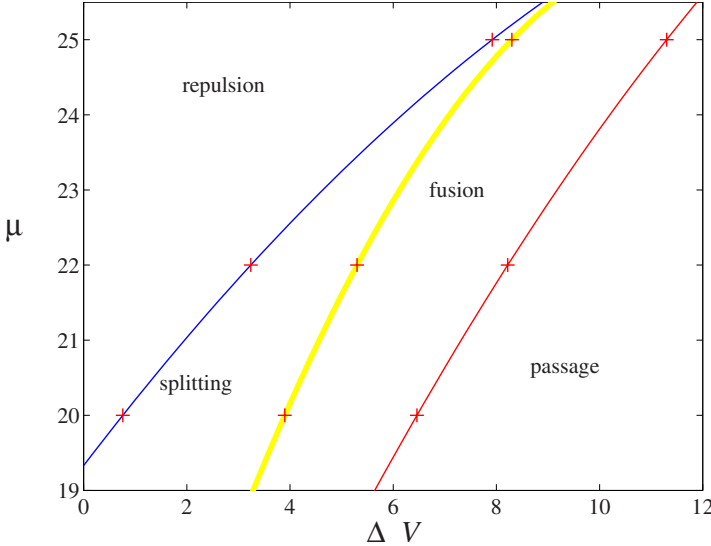

FIG. 5. (Color online) The diagram showing regions of different outcomes of collisions between identical rhombic vortices, with topological charges $S_{1}=S_{2}=1$ and propagation constants $\mu_{1}=\mu_{2} \equiv \mu$, in the plane of the collision velocity, $\Delta V$, and $\mu$. Outcomes called "passage," "fusion," "splitting," and "repulsion" are illustrated by generic examples displayed in Figs. 1-4, respectively.

identical to that displayed in Fig. 5. The only conspicuous difference is observed in the case of the fusion: unlike the situation shown above in Fig. 2, the fusion of vortices with the opposite charges is partial, as illustrated by Fig. 6, which displays trajectories of the centers of fundamental solitons that build the rhombuses and also the full collision picture (cf. caption of Fig. 3). It is concluded from Fig. 6 that four constituent fundamental solitons merge into a single bound state, while four others escape.

\section{Collisions between quadrupoles}

Collisions between identical quadrupoles give rise to outcomes of the same four types as identified above for rhombic vortices. The corresponding diagram is presented in Fig. 7. Similarities and differences in comparison to its counterpart for the rhombuses, which was displayed above in Fig. 5, are obvious from the comparison of the two plots. In particular, it is worthy to note conspicuous differences between relevant

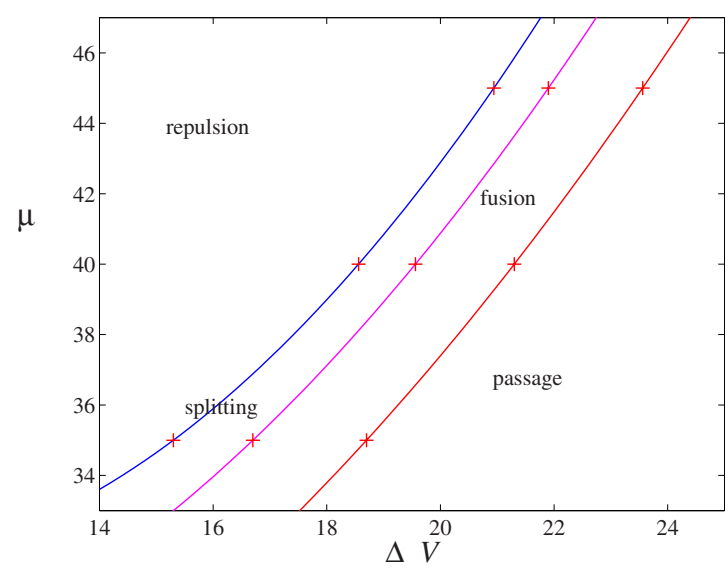

FIG. 7. (Color online) A diagram similar to that in Fig. 5, but showing ranges of different outcomes of collisions between rhombic quadrupoles.

ranges of both parameters, $\Delta V$ and $\mu$, in the two diagrams.

As concerns particular types of the outcomes, a notable difference from the situation considered above is observed in the case of splitting: as shown in Fig. 8, the collision may split the quadrupoles into a set of four two-soliton bound states, which all come to a halt after the interaction ceases, with a weak manifestation of the spontaneous symmetry breaking (cf. Fig. 3).

\section{COLLISIONS OF RHOMBUSES WITH QUADRUPOLES AND FUNDAMENTAL SOLITONS}

Results of collisions between solitons of different types are illustrated below by means of a set of generic examples. Summarizing the results in the form of parameter diagrams would be impractical in this case, as at least three parameters should be taken into regard, viz., two propagation constants (in the case of different types of the solitons, there is no special reason to fix $\mu_{1}=\mu_{2}$ ) and the relative velocity.

Collisions between rhombuses and quadrupoles may give rise to complicated outcomes. For instance, Fig. 9 shows an example of collision-induced splitting that eventually leads
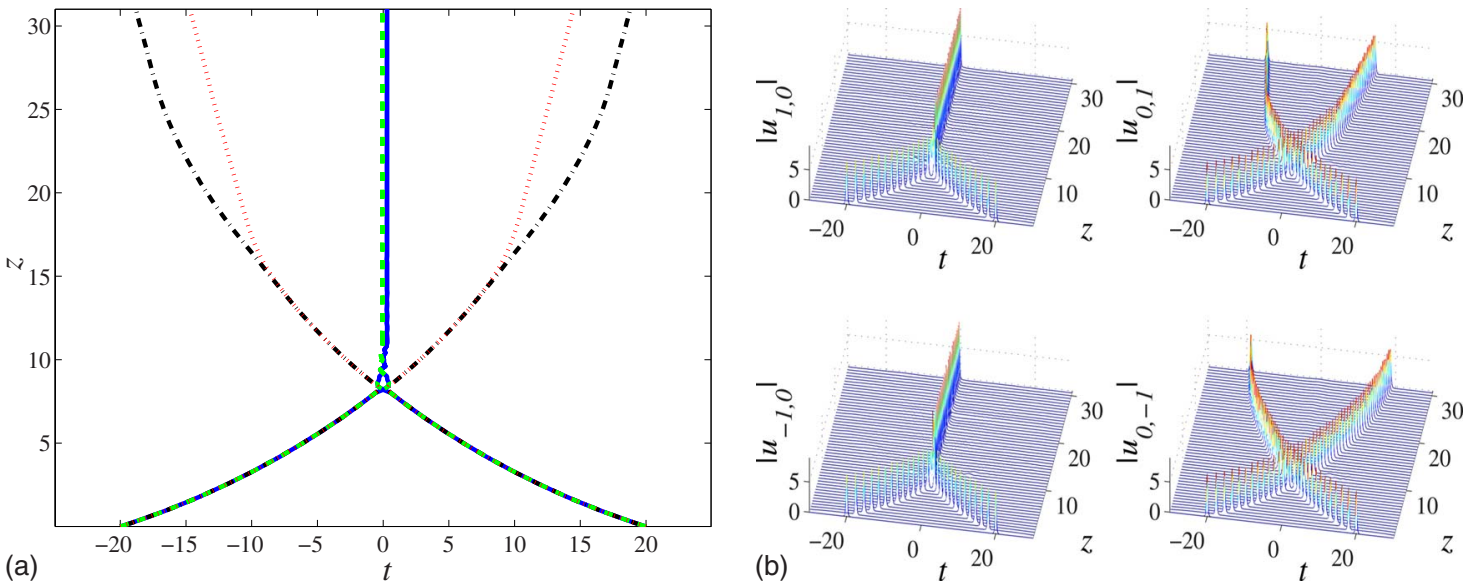

FIG. 6. (Color online) An example of partial fusion of colliding rhombic vortices with opposite topological charges, $S_{1}=-S_{2}=1$, and equal propagation constants, $\mu_{1}=\mu_{2}=22$. The collision velocity is $\Delta V=8$. The panels have the same meaning as in Fig. 3 . 


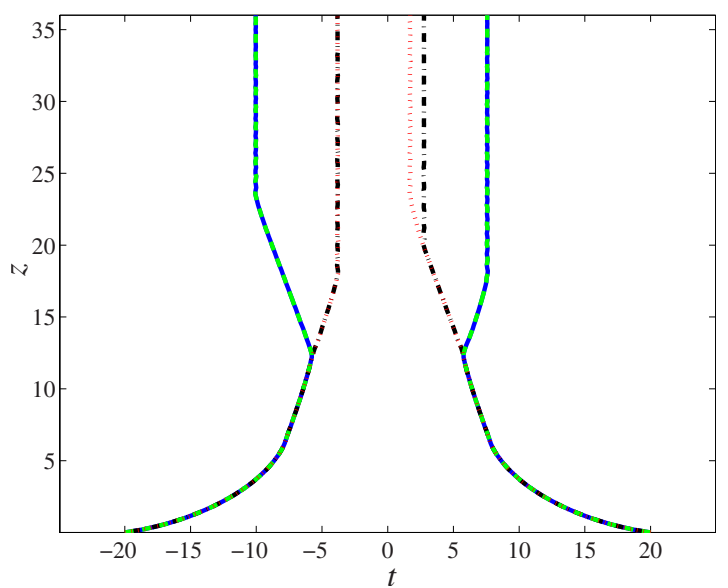

FIG. 8. (Color online) An example of splitting resulting from the collision of two identical rhombic quadrupoles, with propagation constant $\mu=35$ and relative velocity $\Delta V=15.5$.

to appearance of two bound states formed by two pairs of fundamental solitons: one soliton in each pair resulting from the merging of two components [the trajectories of one pair are represented by the two merged red (dotted) and one black (dashed-dotted) lines in the figure, and the other pair includes two merged green (dashed) and one blue (solid) lines], while two other fundamental solitons escape after the collision. Note that both bound states emerge with zero velocities.

The interaction of a free fundamental soliton, launched in the waveguiding core with $(m, n)=(0,0)$, which is nearly empty in the rhombic vortex, with that vortex is stronger than the interactions considered above, as the distance from the central core to those which carry the rhombus is smaller than the distance between vertices of the rhombus. Moreover, the coupling of the central core with the vertices is direct, while the coupling between the vertices is not, as seen from Eq. (1). This strong interaction leads, in most cases, to splitting of the vortex and destruction of its intrinsic phase structure. Even a slowly moving fundamental soliton, which is kept by the repulsion at a large distance from the rhombus, destroys it despite the weakness of the long-range interaction

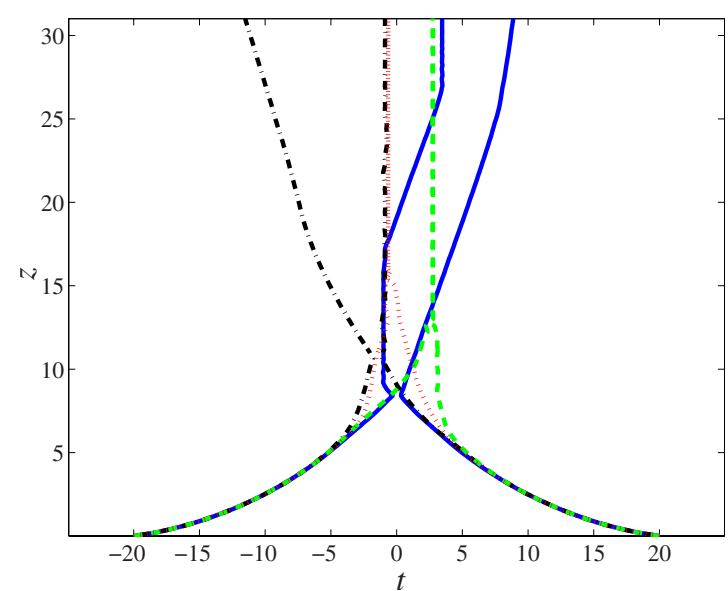

FIG. 9. (Color online) A typical example of splitting resulting from the rhombus-quadrupole collision, with relative velocity $\Delta V$ $=20$. The propagation constant of both complexes prior to the collision is $\mu=40$.

[see Fig. 10(a)]. Panels (b) and (c) in the same figure demonstrate that a faster moving free fundamental soliton bounces from the rhombus and a still faster one gets stopped by it, causing the splitting in any cases. Finally, if the free soliton is very fast, it can pass through the central "hole" of the rhombus quasielastically without destroying it or disturbing its phase structure. For instance, the collision between the rhombus and fundamental soliton, with initial propagation constants $\mu_{\text {rho }}=40$ and $\mu_{\text {sol }}=10$, amounts to the free pass if the relative velocity is $\Delta V=8$ (not shown here in detail), cf. the situation presented in Fig. 10(c), which appertains to the same value of $\Delta V$.

\section{CONCLUSION}

This work aimed to report results of systematic analysis of head-on collisions between various species of stable 3D semidiscrete spatiotemporal soliton complexes in the model of a bundle of nonlinear fiberlike optical waveguides. The same system describes 3D BEC trapped in a deep 2D optical lattice. The model offers the first possibility to study colli-
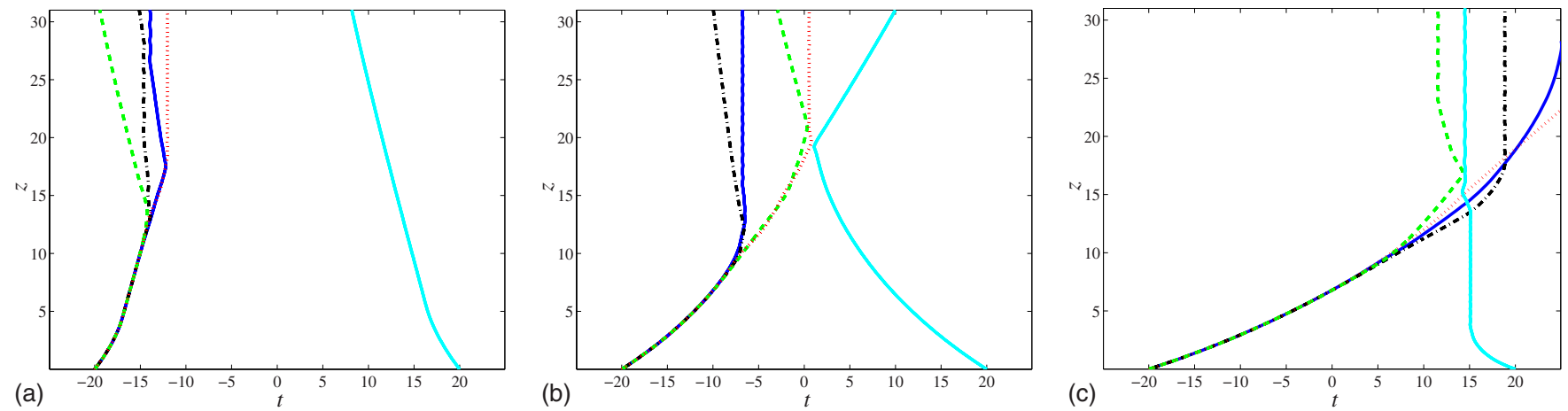

FIG. 10. (Color online) Splitting of a rhombic vortex due to the interaction with a free fundamental soliton, guided by the central core. In the three cases displayed in this figure, the propagation constant of both the rhombus and fundamental soliton before the collision is $\mu$ $=20$, except for the fundamental soliton in panel (c) for which $\mu=30$. The relative velocities are $\Delta V=2,4$, and 8 in panels (a), (b), and (c), respectively. In addition to the graphical notation adopted above (see caption to Fig. 4), in this figure the solid cyan (light gray) line depicts the trajectory of the soliton's center in the central core, with coordinates $(m, n)=(0,0)$. 
sions between 3D vortex solitons in conservative media. We have focused on collisions involving most robust types of semidiscrete solitons, viz., rhombus-shaped vortices with topological charge $S= \pm 1$, and quadrupoles, also of the rhombic type. Fundamental solitons, carried by the central waveguiding core, at which the vortex or quadrupole have the hole, were considered too. Simulations have revealed four outcomes of the collisions: rebound of slowly moving objects, fusion (full or partial fusion, in case of the collision between vortices with equal or opposite charges, respectively), splitting (possibly with the formation of bound states of two solitons), and quasielastic passage of fast solitons through each other. Full parameter diagrams have been produced for collisions between identical vortices and quadru- poles. The rhombic vortex was found to be especially vulnerable to splitting due to the collision with a free fundamental soliton moving in the central core.

The analysis presented in this work can be extended in various directions. One of them is the consideration of interactions between solitons in the model of bundles with a triangular or hexagonal transverse structure, cf. Ref. [17], where $2 \mathrm{D}$ discrete solitons were constructed in similar settings. As concerns the existence of vortex solitons and collisions between them, an especially interesting generalization of the present model may be one including twist of the waveguiding bundle. In the 2D version of the twisted model, spatial discrete fundamental solitons and solitary vortices were reported in Ref. [43].
[1] D. N. Christodoulides, F. Lederer, and Y. Silberberg, Nature (London) 424, 817 (2003); F. Lederer, G. I. Stegeman, D. N. Christodoulides, G. Assanto, M. Segev, and Y. Silberberg, Phys. Rep. 463, 1 (2008); S. Flach and A. V. Gorbach, ibid. 467, 1 (2008); Y. S. Kivshar, Laser Phys. Lett. 5, 703 (2008).

[2] P. G. Kevrekidis, K. Ø. Rasmussen, and A. R. Bishop, Int. J. Mod. Phys. B 15, 2833 (2001).

[3] D. N. Christodoulides and R. I. Joseph, Opt. Lett. 13, 794 (1988).

[4] A. Trombettoni and A. Smerzi, Phys. Rev. Lett. 86, 2353 (2001); G. L. Alfimov, P. G. Kevrekidis, V. V. Konotop, and M. Salerno, Phys. Rev. E 66, 046608 (2002); R. CarreteroGonzalez and K. Promislow, Phys. Rev. A 66, 033610 (2002); F. S. Cataliotti, S. Burger, C. Fort, P. Maddaloni, F. Minardi, A. Trombettoni, A. Smerzi, and M. Inguscio, Science 293, 843 (2001); M. Greiner, O. Mandel, T. Esslinger, T. W. Hänsch, and I. Bloch, Nature (London) 415, 39 (2002); N. K. Efremidis and D. N. Christodoulides, Phys. Rev. A 67, 063608 (2003).

[5] M. A. Porter, R. Carretero-González, P. G. Kevrekidis, and B. A. Malomed, Chaos 15, 015115 (2005).

[6] J. E. Heebner and R. W. Boyd, J. Mod. Opt. 49, 2629 (2002); P. Chak, J. E. Sipe, and S. Pereira, Opt. Lett. 28, 1966 (2003); J. J. Baumberg, P. G. Savvidis, R. M. Stevenson, A. I. Tartakovskii, M. S. Skolnick, D. M. Whittaker, and J. S. Roberts, Phys. Rev. B 62, R16247 (2000); P. G. Savvidis and P. G. Lagoudakis, Semicond. Sci. Technol. 18, S311 (2003).

[7] H. S. Eisenberg, Y. Silberberg, R. Morandotti, A. R. Boyd, and J. S. Aitchison, Phys. Rev. Lett. 81, 3383 (1998).

[8] T. Pertsch, U. Peschel, J. Kobelke, K. Schuster, H. Bartelt, S. Nolte, A. Tünnermann, and F. Lederer, Phys. Rev. Lett. 93, 053901 (2004).

[9] N. K. Efremidis, S. Sears, D. N. Christodoulides, J. W. Fleischer, and M. Segev, Phys. Rev. E 66, 046602 (2002); N. K. Efremidis, J. Hudock, D. N. Christodoulides, J. W. Fleischer, O. Cohen, and M. Segev, Phys. Rev. Lett. 91, 213906 (2003); J. W. Fleischer, G. Bartal, O. Cohen, T. Schwartz, O. Manela, B. Freedman, M. Segev, H. Buljan, and N. K. Efremidis, Opt. Express 13, 1780 (2005).

[10] J. W. Fleischer, M. Segev, N. K. Efremidis, and D. N. Christodoulides, Nature (London) 422, 147 (2003); J. W.
Fleischer, G. Bartal, O. Cohen, O. Manela, M. Segev, J. Hudock, and D. N. Christodoulides, Phys. Rev. Lett. 92, 123904 (2004).

[11] B. A. Malomed and P. G. Kevrekidis, Phys. Rev. E 64, 026601 (2001).

[12] D. N. Neshev, T. J. Alexander, E. A. Ostrovskaya, Y. S. Kivshar, H. Martin, I. Makasyuk, and Z. Chen, Phys. Rev. Lett. 92, 123903 (2004); J. W. Fleischer, G. Bartal, O. Cohen, O. Manela, M. Segev, J. Hudock, and D. N. Christodoulides, ibid. 92, 123904 (2004).

[13] P. G. Kevrekidis, B. A. Malomed, Z. Chen, and D. J. Frantzeskakis, Phys. Rev. E 70, 056612 (2004).

[14] H. Sakaguchi and B. A. Malomed, Europhys. Lett. 72, 698 (2005); R. Driben and B. A. Malomed, Eur. Phys. J. D 50, 317 (2008).

[15] O. Manela, O. Cohen, G. Bartal, J. W. Fleischer, and M. Segev, Opt. Lett. 29, 2049 (2004).

[16] J. Yang, I. Makasyuk, P. G. Kevrekidis, H. Martin, B. A. Malomed, D. J. Frantzeskakis, and Z. Chen, Phys. Rev. Lett. 94, 113902 (2005).

[17] P. G. Kevrekidis, B. A. Malomed, and Y. B. Gaididei, Phys. Rev. E 66, 016609 (2002); C. R. Rosberg, D. N. Neshev, A. A. Sukhorukov, W. Królikowski, and Y. S. Kivshar, Opt. Lett. 32, 397 (2007); T. J. Alexander, A. S. Desyatnikov, and Y. S. Kivshar, ibid. 32, 1293 (2007); B. Terhalle, T. Richter, A. S. Desyatnikov, D. N. Neshev, W. Królikowski, F. Kaiser, C. Denz, and Y. S. Kivshar, Phys. Rev. Lett. 101, 013903 (2008); K. J. H. Law, H. Susanto, and P. G. Kevrekidis, Phys. Rev. A 78, 033802 (2008).

[18] N. C. Panoiu, R. M. Osgood, and B. A. Malomed, Opt. Lett. 31, 1097 (2006); N. C. Panoiu, B. A. Malomed, and R. M. Osgood, Phys. Rev. A 78, 013801 (2008).

[19] M. J. Ablowitz, Z. H. Musslimani, and G. Biondini, Phys. Rev. E 65, 026602 (2002).

[20] I. E. Papacharalampous, P. G. Kevrekidis, B. A. Malomed, and D. J. Frantzeskakis, Phys. Rev. E 68, 046604 (2003).

[21] J. Meier, G. I. Stegeman, Y. Silberberg, R. Morandotti, and J. S. Aitchison, Phys. Rev. Lett. 93, 093903 (2004); J. Meier, G. I. Stegeman, D. N. Christodoulides, R. Morandotti, M. Sorel, H. Yang, G. Salamo, J. S. Aitchison, and Y. Silberberg, Opt. Express 13, 1797 (2005); Y. Linzon, Y. Sivan, B. Malomed, 
M. Zaezjev, R. Morandotti, and S. Bar-Ad, Phys. Rev. Lett. 97, 193901 (2006).

[22] D. Cheskis, S. Bar-Ad, R. Morandotti, J. S. Aitchison, H. S. Eisenberg, Y. Silberberg, and D. Ross, Phys. Rev. Lett. 91, 223901 (2003).

[23] A. Szameit, J. Burghoff, T. Pertsch, S. Nolte, A. Tünnermann, and F. Lederer, Opt. Express 14, 6055 (2006); A. Szameit, T. Pertsch, F. Dreisow, S. Nolte, A. Tünnermann, U. Peschel, and F. Lederer, Phys. Rev. A 75, 053814 (2007).

[24] T. Pertsch, U. Peschel, F. Lederer, J. Burghoff, M. Will, S. Nolte, and A. Tünnermann, Opt. Lett. 29, 468 (2004); A. Szameit, D. Blömer, J. Burghoff, T. Schreiber, T. Pertsch, S. Nolte, A. Tünnermann, and F. Lederer, Opt. Express 13, 10552 (2005).

[25] P. Xie, Z.-Q. Zhang, and X. Zhang, Phys. Rev. E 67, 026607 (2003); A. Ferrando, M. Zacarés, P. F. de Cordoba, D. Binosi, and J. A. Monsoriu, Opt. Express 11, 452 (2003); 12, 817 (2004); A. Ferrando, M. Zacarés, and M. A. García-March, Phys. Rev. Lett. 95, 043901 (2005).

[26] B. A. Malomed, D. Mihalache, F. Wise, and L. Torner, J. Opt. B: Quantum Semiclassical Opt. 7, R53 (2005).

[27] A. B. Aceves, C. De Angelis, A. M. Rubenchik, and S. K. Turitsyn, Opt. Lett. 19, 329 (1994); A. V. Buryak and N. N. Akhmediev, IEEE J. Quantum Electron. 31, 682 (1995).

[28] C. J. Benton, A. V. Gorbach, and D. V. Skryabin, Phys. Rev. A 78, 033818 (2008).

[29] A. B. Aceves, G. G. Luther, C. De Angelis, A. M. Rubenchik, and S. K. Turitsyn, Phys. Rev. Lett. 75, 73 (1995).

[30] A. B. Aceves, C. De Angelis, T. Peschel, R. Muschall, F. Lederer, S. Trillo, and S. Wabnitz, Phys. Rev. E 53, 1172 (1996); A. B. Aceves and M. Santagiustina, ibid. 56, 1113 (1997).

[31] A. B. Aceves, C. De Angelis, G. G. Luther, and A. M. Rubenchik, Opt. Lett. 19, 1186 (1994); E. W. Laedke, K. H. Spatschek, S. K. Turitsyn, and V. K. Mezentsev, Phys. Rev. E 52, 5549 (1995); S. Darmanyan, I. Relke, and F. Lederer, ibid. 55, 7662 (1997); A. B. Aceves, M. Santagiustina, and C. De
Angelis, J. Opt. Soc. Am. B 14, 1807 (1997); I. Relke, Phys. Rev. E 57, 6105 (1998); M. Stepić, L. Hadžievski, and M. M. Skorić, ibid. 65, 026604 (2002); A. V. Yulin, D. V. Skryabin, and A. G. Vladimirov, Opt. Express 14, 12347 (2006).

[32] Z. Y. Xu, Y. V. Kartashov, L. C. Crasovan, D. Mihalache, and L. Torner, Phys. Rev. E 70, 066618 (2004).

[33] D. Mihalache, D. Mazilu, F. Lederer, and Y. S. Kivshar, Opt. Express 15, 589 (2007); D. Mihalache, D. Mazilu, Y. S. Kivshar, and F. Lederer, ibid. 15, 10718 (2007); D. Mihalache, D. Mazilu, F. Lederer, and Y. S. Kivshar, Opt. Lett. 32, 3173 (2007); D. Mihalache, D. Mazilu, F. Lederer, and Y. S. Kivshar, Phys. Rev. A 79, 013811 (2009).

[34] D. Mihalache, D. Mazilu, F. Lederer, and Y. S. Kivshar, Opt. Lett. 32, 2091 (2007).

[35] M. I. Molina and Y. S. Kivshar, Phys. Lett. A 362, 280 (2007).

[36] B. B. Baizakov, B. A. Malomed, and M. Salerno, Phys. Rev. A 70, 053613 (2004); D. Mihalache, D. Mazilu, F. Lederer, Y. V. Kartashov, L.-C. Crasovan, and L. Torner, Phys. Rev. E 70, 055603(R) (2004).

[37] H. Leblond, B. A. Malomed, and D. Mihalache, Phys. Rev. A 77, 063804 (2008).

[38] H. Leblond, B. A. Malomed, and D. Mihalache, Phys. Rev. E 76, 026604 (2007).

[39] P. G. Kevrekidis, B. A. Malomed, D. J. Frantzeskakis, and R. Carretero-González, Phys. Rev. Lett. 93, 080403 (2004); R. Carretero-González, P. G. Kevrekidis, B. A. Malomed and D. J. Frantzeskakis, ibid. 94, 203901 (2005).

[40] D. Mihalache, D. Mazilu, F. Lederer, H. Leblond, and B. A. Malomed, Phys. Rev. A 77, 033817 (2008).

[41] D. Mihalache, D. Mazilu, F. Lederer, H. Leblond, and B. A. Malomed, Phys. Rev. E 78, 056601 (2008).

[42] G. P. Agrawal, Nonlinear Fiber Optics (Academic, San Diego, 1995).

[43] J. Cuevas, B. A. Malomed, and P. G. Kevrekidis, Phys. Rev. E 76, 046608 (2007). 